\title{
First Lasing of FLASH2 at DESY
}

The free-electron laser FLASH at DESY in Hamburg, Germany, has been extended by a second undulator line in a separate tunnel and a new experimental hall (see Fig. 1). The existing undulator line, called FLASH1, and the new one, which includes provisions for external seeding to be realized later, called FLASH2, use both the same linear accelerator. They are operated in tandem by separating the pulse train into two sections which are then directed to the different undulators. The linac energy is determined by the needs of the experiments at FLASH1, whereas the wavelength at FLASH2 can be varied in the limits of the tuning range of the undulators.

The mounting of the new electron beamline, including 12 tunable undulator modules, was finished in January 2014. The commissioning is done in parallel to FLASH1 operations and started in March 2014. On August 20, 2014, first lasing at FLASH2 was observed at a wavelength of about $40 \mathrm{~nm}$ shortly after closing the last four undulator modules. The linac was being operated at an energy of $680 \mathrm{MeV}$. A few days later, the first photon spectrum could be measured with two different undulator gap settings, providing photon wavelengths of $42 \mathrm{~nm}$ and $23.5 \mathrm{~nm}$, respectively. The opening angle of the measured radiation cone of $80 \mu \mathrm{rad}$ as well as the intensity fluctuation statistics agree with theoretical expectations for SelfAmplified Spontaneous Emission (SASE) mode (see Fig. 2). Further optimization is under way.

It is noteworthy that the first lasing of FLASH2 has been achieved while FLASH1 was running in SASE with 250 bunches in a bunch train at a wavelength of $13.5 \mathrm{~nm}$. FLASH is the worldwide first free-electron laser facility for soft X-rays where the many electron bunches, which only linear accelerators using superconducting RF technology can provide, are distributed simultaneously to two independent FELs with the repetition rate of the accelerator.

In practice the capacity of FLASH for users will be doubled. The successful operation of both undulator lines also supports the multi-beamline concept pursued at the European XFEL facility.

Authors: Bart Faatz and Siegfried Schreiber (DESY Hamburg, Germany)
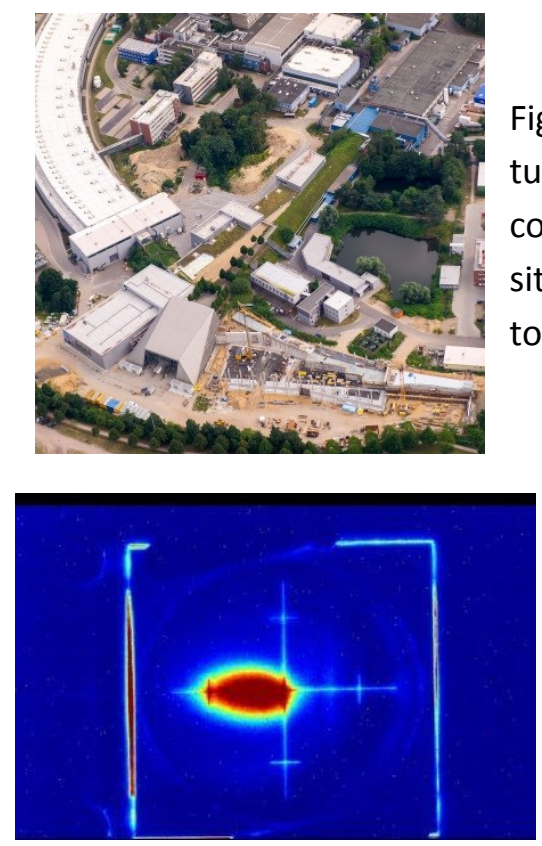

Fig. 1: Aerial View of the entire FLASH facility at DESY including the new tunnel (on the left side) and the new experimental hall (in the lower left corner) in July 2014. The curved PETRA III hall (left) and the construction site of the PETRA extension North (in the lower right corner) are adjacent to the two FLASH experimental halls.

Fig. 2: Cross section of the SASE beam measured with Ce:YAG screen $18 \mathrm{~m}$ behind the undulator. The opening angle of the radiation cone is $80 \mu \mathrm{rad}$, the radiation cone for spontaneous radiation is about 15 times wider. 